Thomas Kemper \& Janka Goldan

\title{
Analysen zur Entwicklung der schulischen Inklusion Potenziale von Daten der amtlichen Schulstatistik am Beispiel von Nordrhein-Westfalen
}

\section{Zusammenfassung}

Basierend auf Daten der amtlichen Schulstatistik wird exemplarisch für ein Bundesland anhand von inklusionsrelevanten Kennzahlen die Entwicklung der schulischen Inklusion überprüft. Für die Schuljahre 2012/13 bis 2016/17 wird untersucht, wie sich die Anzahl der Schüler*innen mit sonderpädagogischem Förderbedarf entwickelt hat und wie viele hiervon an Schulformen der Allgemeinen Schule und an Förderschulen lernen. Darüber hinaus wird mit der GU-Quote eine neue Kennzahl eingeführt, die abbildet, wie sich der Anteil der Schulen, die Inklusion im Sinne der Operationalisierung durchführen, entwickelt. Weiter werden die zwischen den Kommunen bestehenden erheblichen regionalen Disparitäten hinsichtlich inklusionsrelevanter Kennzahlen aufgezeigt.

Schlüsselwörter: schulische Inklusion, amtliche Schulstatistik, sonderpädagogischer Förderbedarf, Gemeinsamer Unterricht, regionale Disparitäten

\section{Analyzing the Development of Inclusive Education}

The Analytical Potential of Official School Statistic Data, Using the Example of North Rhine-Westphalia

\begin{abstract}
On the basis of data from official school statistics, the development of inclusive education is examined for one federal state with regard to inclusion-relevant key figures. For the school years 2012/13 to 2016/17, the number of students with special educational needs is analyzed and whether they are taught in different general or special schools. In addition, a new indicator, the GU-quota [quota of common learning], is introduced, which shows the quota of schools providing inclusive education. Furthermore, it is referred to the considerable regional disparities which exist between the municipalities in terms of the different quotas.
\end{abstract}

Keywords: inclusive schooling, inclusive education, official school statistics, special educational needs, regional disparities 


\section{Einleitung}

Am 26. März 2009 wurde das Übereinkommen der Vereinten Nationen über die Rechte von Menschen mit Behinderung (UN-BRK) im deutschen Bundestag ratifiziert. Die schulische Inklusion, d.h. das Gemeinsame Lernen von Schüler*innen mit und ohne Behinderung, soll nach Artikel 24 der UN-BRK den gesetzlichen Regelfall bilden und nicht mehr die Ausnahme sein.

Obwohl die UN-BRK seit den Allgemeinen Bemerkungen Nr. 4 des UN-Ausschusses für die Rechte von Menschen mit Behinderungen keinen Raum für Interpretation lässt, herrscht politisch Uneinigkeit darüber, ob die Schulform Förderschule aufgegeben werden muss (Aichele \& Kroworsch, 2017; Kroworsch, 2017). Unstrittig ist, dass die UN-BRK verlangt, den Anteil der Schüler*innen mit sonderpädagogischem Förderbedarf an Allgemeinen Schulen deutlich zu steigern, während die Anzahl der separiert an Förderschulen unterrichteten Schüler*innen sinken muss. Der gleichberechtigte Zugang zu einem allgemeinen Bildungssystem schließt zudem das Recht auf wohnortnahe Beschulung mit ein (Ziegeler \& Schubert, 2015). Langfristig ist daher zu erwarten, dass die Anzahl der Förderschulen sinkt und die Anzahl der Schulen, an denen Gemeinsames Lernen praktiziert wird, steigt.

Inzwischen haben zwar alle Landesregierungen die UN-BRK in ihre Schulgesetze aufgenommen. Die gesetzliche Umsetzung ist jedoch so heterogen, dass es sehr schwierig ist, die Bundesländer hinsichtlich der zu erwartenden Entwicklungen miteinander zu vergleichen (Hillenbrand, 2013; Klemm, 2014; Malecki, 2014; Mißling \& Ückert, 2014). Auch hatten die Länder hinsichtlich des Gemeinsamen Lernens verschiedene Ausgangsbedingungen.

Um die Entwicklung der schulischen Inklusion beurteilen zu können, wird diese auf Länderebene im Verlauf von mehreren Schuljahren (bzw. in einer Zeitreihe) betrachtet. Der vorliegende Beitrag untersucht mittels einer schulstatistischen Analyse am Beispiel von Nordrhein-Westfalen (NRW), ob und in welchem Umfang regionale Disparitäten auf Ebene der Kreise und kreisfreien Städte bestehen. Um die Frage beantworten zu können, ob Inklusion im Sinne des Rechts auf wohnortnahe Beschulung flächendeckend fortschreitet, wird eine neue Kennzahl eingeführt - die sogenannte GU-Quote.

\section{Forschungsstand und Hintergrund}

Zunächst werden kurz die Kennzahlen definiert, die zur wissenschaftlichen Einschätzung des Standes der Inklusion regelmäßig berichtet werden; anschließend wird der aktuelle Stand der Forschung hinsichtlich schulstatistischer Inklusionsanalysen aufgearbeitet. 
Unter dem Inklusionsanteil wird nach Klemm (2013) der Anteil inklusiv unterrichteter Schülerinnen an allen Schüler*innen mit sonderpädagogischem Förderbedarf verstanden. Komplementär dazu wird der Exklusionsanteil berichtet. Hierbei handelt es sich um den Anteil separiert in Förderschulen unterrichteter Schüler*innen an allen Schüler ${ }^{\star}$ innen mit sonderpädagogischem Förderbedarf (vgl. ebd.). Z. B. lag der Inklusionsanteil in Niedersachsen im Schuljahr 2008/09 bei 6,6 Prozent; der Exklusionsanteil betrug 93,4 Prozent (Klemm, 2014).

Daneben werden häufig die Exklusions- und seltener auch die Inklusionsquote angeführt. Unter der Exklusionsquote wird der Anteil der „Förderschüler“ (ebd.) an allen Schüler ${ }^{\star}$ innen im schulpflichtigen Alter verstanden. ${ }^{1}$ Diese Quote lag im Schuljahr 2008/09 in NRW bei 5,2 Prozent und ist bis zum Schuljahr 2012/13 unverändert geblieben (vgl. ebd.). Die Exklusionsquote für NRW lag im Schuljahr 2012/13 über dem Bundesdurchschnitt von 4,8 Prozent, der seit dem Schuljahr 2000/01 sogar leicht angestiegen ist (Autorengruppe Bildungsberichterstattung, 2014, S. 178). Eine im Zeitverlauf sinkende Exklusionsquote gilt als Indiz für zunehmende schulische Inklusion, wobei die Gesamtschülerzahl bei der Beurteilung immer berücksichtigt werden sollte. Gleiches gilt für die Inklusionsquote, die den Anteil der Schüler*innen mit sonderpädagogischem Förderbedarf an Allgemeinen Schulen bezogen auf alle Schüler*innen angibt (Klemm, 2014).

Um die genannten Kennzahlen sinnvoll interpretieren zu können, muss gleichzeitig auch die Förderquote in den Blick genommen werden. Unter der Förderquote wird der Anteil der Schülerinnen mit diagnostiziertem sonderpädagogischem Förderbedarf an allen Schüler*innen im schulpflichtigen Alter verstanden. Dabei spielt es keine Rolle, ob diese eine Allgemeine Schule oder eine Förderschule besuchen (Klemm, 2013). Z.B. haben zwar die Daten der Schulstatistik in NRW für die letzten Schuljahre gezeigt, dass der Inklusionsanteil kontinuierlich ansteigt. Dieser Trend - der auch für Deutschland insgesamt festzustellen ist - muss allerdings relativiert werden, da trotz Berücksichtigung des Bevölkerungsrückgangs auch die Förderquote stetig steigt und die Anzahl der Förderschüler*innen allenfalls nur leicht zurückgeht (Autorengruppe Bildungsberichterstattung, 2014; Hollenbach-Biele, 2014; Klemm, 2014, 2015; KMK, 2016). In diesem Zusammenhang wird zu Recht die Frage aufgeworfen, ob zunehmende schulische Inklusion oder die Anzahl der Diagnosen an Regelschulen dafür verantwortlich sind, dass der Inklusionsanteil steigt (Klemm, 2014, 2015).

In der wissenschaftlichen Literatur werden primär die genannten Kennzahlen verwendet um zu beurteilen, wie sich die schulische Inklusion entwickelt. Häufig er-

1 Vereinzelt finden sich abweichende Bezeichnungen. Die Exklusionsquote wird z.T. auch als Förderschulbesuchs- oder Separationsquote bezeichnet (vgl. z. B. Brüggemann \& Tegge, 2016). Eine Auseinandersetzung mit den unterschiedlichen Begrifflichkeiten kann in diesem Rahmen nicht erfolgen; nachfolgend werden die gängigen Definitionen verwendet. 
folgt noch eine Differenzierung nach Art des sonderpädagogischen Förderbedarfs, nach der Schulform sowie nach der Primar- und Sekundarstufe. ${ }^{2}$ Aktuelle schulstatistische Analysen beziehen sich vor allem auf Gegenüberstellungen der 16 Länder im Verlauf der letzten Schuljahre (Hollenbach-Biele, 2014; Klemm \& Preuss-Lausitz, 2017; Klemm, 2010, 2013, 2014, 2015; KMK, 2016; Malecki, 2014). Die Befunde zeigen auch für die anderen Länder steigende Inklusionsanteile bei kaum rückläufigen Exklusionsquoten (Döttinger \& Hollenbach-Biele, 2015; Hollenbach-Biele, 2014; Klemm, 2013, 2015). Wie Lütje-Klose, Neumann und Streese (2017) für NRW herausstellten, ist auch die Anzahl der Förderschulen in den letzten Jahren stabil geblieben. Lediglich im Förderschwerpunkt Lernen ist die Anzahl der Förderschulen zurückgegangen (ebd.).

Im Ländervergleich zeichnen die Kennzahlen darüber hinaus ein äußerst heterogenes Bild, insbesondere hinsichtlich divergierender Förderquoten, die auf unterschiedliche Praxen bei der Diagnose sonderpädagogischer Förderbedarfe zurückgeführt werden (Autorengruppe Bildungsberichterstattung, 2014; Hillenbrand, 2013; Klemm, 2015; Sälzer, Gebhardt, Müller \& Pauly, 2015). Neben den unterschiedlichen Ausgangs- und Gesetzeslagen ist dies der Hauptgrund, warum die Kennzahlen der Länder nur bedingt miteinander verglichen werden können (z. B. Klemm, 2014, 2015).

Das Problem der Vergleichbarkeit beschränkt sich bei genauer Betrachtung nicht nur auf die Landesebene. Das Kommunalrecht sieht vor, dass die Kreise, kreisfreien Städte und Gemeinden als Schulträger für die äußeren Schulangelegenheiten zuständig sind. Dies führt dazu, dass sie eigenständige politische Entscheidungen treffen (Klemm \& Preuss-Lausitz, 2017). Daher sind kleinräumige Analysen von besonderem Interesse. Studien, die diesen Gedanken aufnehmen und Disparitäten bis auf die Ebene der Kreise und kreisfreien Städte aufzeigen, liegen z. B. von Kemper (2012) sowie Kemper und Weishaupt (2011) vor. Anhand schulstatistischer Analysen für NRW und Rheinland-Pfalz konnten erhebliche regionale Unterschiede in der Förderquote und der Förderschulbesuchsquote u.a. von ausländischen im Vergleich zu deutschen Schüler ${ }^{\star}$ innen festgestellt werden. Eine aktuelle Studie aus Bayern (Heimlich \& Wittko, 2018) zeigt ebenfalls erhebliche regionale Unterschiede bei der schulischen Inklusion. Insbesondere die Förderquote streut in den Landkreisen von 4,3 bis 28,8 Prozent. Obgleich diese Disparitäten zu einem erheblichen Teil auf die ungleiche regionale Verteilung von Förderschulstandorten zurückzuführen sind, müssen weitere Untersuchungen klären, inwieweit Unterschiede in der Diagnose- und Überweisungspraxis tatsächlich eine Rolle spielen. Weitere Analysen legen einen Schwerpunkt auf Schülerinnen an Förderschulen differenziert nach sonderpädago-

2 Aktuelle Untersuchungen beschränken sich i.d.R. auf eine Differenzierung zwischen Primar- und Sekundarstufe, weil auch hier eine Vergleichbarkeit zwischen den Ländern aufgrund unterschiedlicher Schulformen und Schulbesuchsdauer nur bedingt gegeben ist. Da sich der empirische Teil des vorliegenden Beitrags beispielhaft auf das Land NRW bezieht, ist eine Differenzierung nach Schulformen möglich. 
gischen Förderschwerpunkten (vgl. z. B. Weishaupt, 2017, für Hessen, Niedersachsen und Rheinland-Pfalz). Andere regionale Analysen zur schulischen Inklusion liegen von Dietze (2011) für die Bundesrepublik Deutschland, von Klemm (2010) für Berlin und binär differenziert nach Kreisen und kreisfreien Städten von Weishaupt (2018) für Hessen und Rheinland-Pfalz vor. Diese sind insbesondere für Fragen kommunaler Bildungsplanung (z. B. bei dezentraler Steuerung von Ressourcen und Schulentwicklungsplanung) von Bedeutung. Für den Bereich der Bildungsforschung tangieren diese Unterschiede u.a. Aspekte der Bildungsgerechtigkeit, die aufgrund der dezentralen Strukturen des deutschen Bildungssystems zwischen den einzelnen Schulamtsbezirken unterschiedlich gewährleistet sein kann (dies betrifft z. B. Unterschiede in der Feststellung von sonderpädagogischen Förderbedarfen, für die Schulämter zuständig sind).

\section{Forschungsfragen und Methode}

Um nachfolgend die regionalen und schulformspezifischen Unterschiede in der Entwicklung der schulischen Inklusion exemplarisch für ein Land und innerhalb dessen darzustellen, werden Daten der amtlichen Schulstatistik für das Bundesland NRW sekundäranalytisch ausgewertet. Hierzu werden die zuvor dargestellten Kennzahlen für die Schuljahre 2012/13 bis einschließlich 2016/17 berichtet und analysiert. In der Literatur blieb ein wichtiges Merkmal schulischer Inklusion bislang unbeachtet, nämlich die Anzahl der Schulen, auf die sich die inklusiv unterrichteten Schüler*innen verteilen. Aus dieser Zahl lässt sich die folgende Forschungsfrage ableiten: Findet Gemeinsames Lernen in nur wenigen Schulen statt (Separation im Allgemeinen Schulsystem) oder wird es - im Sinne eines „integrativen Bildungssystems“ nach Art. 24 Abs. 1 UN-BRK - flächendeckend eingerichtet? Zur Beantwortung dieser Frage soll eine Kennzahl ergänzt werden, die zusätzliche Informationen zur Entwicklung einer inklusiven Schullandschaft liefert. Berechnet wird der Anteil der Schulen, an denen Gemeinsamer Unterricht (GU) stattfindet, bezogen auf alle Allgemeinbildenden Schulen. Dies wird wie folgt operationalisiert: GU liegt an einer Allgemeinen Schule dann vor, wenn mindestens eine Schülerin bzw. ein Schüler mit festgestelltem sonderpädagogischem Förderbedarf diese Schule besucht. Der Anteil der Schulen mit GU an allen Allgemeinen Schulen einer Region wird als Anteil des Gemeinsamen Unterrichts berichtet, den wir nachfolgend als „GU-Quote“ bezeichnen. ${ }^{3}$

Damit wäre neben dem Inklusionsanteil und der Inklusionsquote eine Kennzahl gegeben, die einerseits Aufschluss darüber gibt, auf wie viele Schulen sich die Anzahl der inklusiv unterrichteten Schülerinnen verteilt. Andererseits lässt eine GU-Quote

3 Eine ähnliche, inhaltlich nicht näher erläuterte Kennzahl („Anteil der allgemeinbildenden Schulen [...], die Schülerinnen und Schüler mit sonderpädagogischer Förderung unterrichten") wurde nur in Autorengruppe Bildungsberichterstattung (2018, S. 106) gefunden. 
im Sinne des in der UN-BRK verankerten Rechts auf wohnortnahe Beschulung Rückschlüsse darauf zu, inwieweit dieses Recht bereits verwirklicht ist, d.h., wie viele Schulen sich bereits der schulischen Inklusion geöffnet haben.

Im Folgenden sollen die inklusionsrelevanten Kennzahlen einschließlich der GUQuote analysiert und regionale Disparitäten kartografisch dargestellt werden.

\section{Ergebnisse}

Die Anzahl Allgemeiner Schulen geht in NRW zwischen 2012/13 und 2016/17 um insgesamt 261 Schulen bzw. um 5 Prozent zurück (von 5.141 auf 4.880 Schulen; vgl. Tab. 1). ${ }^{4}$ Differenziert nach Schulform fallen die Entwicklungen unterschiedlich aus. Die stärksten Rückgänge sind für Grund-, Haupt- und Gemeinschaftsschulen zu beobachten. Demgegenüber steigt die Anzahl der Gesamtschulen um knapp ein Drittel, während sich die Anzahl der Sekundarschulen sogar beinahe verdreifacht.

Zudem geht im Untersuchungszeitraum die Anzahl der Förderschulen um insgesamt 189 Schulen zurück. Die deutlichsten Rückgänge sind erwartungsgemäß nach 2014/15 zu beobachten, da das 9. Schulrechtsänderungsgesetz (9. SchRÄG) zum Schuljahr 2014/15 in NRW in Kraft trat und die schulische Inklusion damit zum gesetzlichen Regelfall wurde. Zwar haben Eltern immer noch das Recht, eine Förderschule als Förderort für ihr Kind zu wählen. Seit 2013 gibt es allerdings eine verschärfte Mindestgrößenverordnung für Förderschulen, die zu einem Rückgang der Schulform beigetragen hat und die bei der Interpretation der Zahlen $\mathrm{zu}$ berücksichtigen ist. ${ }^{5}$ Zudem wurde gezeigt, dass in NRW die Kreise z. T. dazu übergehen, Förderzentren zu bilden (Schneider, Klemm, Kemper \& Goldan, 2017). Insgesamt sinkt zwar die Anzahl der Förderschulen um 26,8 Prozent; die Entwicklung der Anzahl der Förderschüler*innen geht jedoch weniger deutlich zurück - und zwar um nur 14,1 Prozent (eigene Berechnung, ohne Abbildung). Zudem ist bekannt, dass sich dieser Rückgang in erster Linie auf den Förderschwerpunkt Lernen bezieht (vgl. Lütje-Klose et al., 2017). Dieser Befund kann für das Schuljahr 2016/17 fortgeschrieben werden: Neben einem erheblichen Rückgang im Förderschwerpunkt Lernen um 23,7 Prozent ist es zwischen den Schuljahren 2012/13 und 2016/17 lediglich zu einem marginalen Rückgang im Förderschwerpunkt körperliche und motorische Entwicklung gekommen (-1\%); für die anderen Förderschwerpunkte sind stagnierende bis leicht zunehmende Schülerzahlen festzustellen (Hören und Kommunikation $(\mathrm{HK})+0,5 \%$,

4 Diese Entwicklung steht im Zusammenhang mit sinkenden Schülerzahlen innerhalb desselben Zeitraumes - und zwar um insgesamt 5,6 Prozent von 1,96 Mio. auf 1,85 Mio. (eigene Berechnung, ohne Abbildung).

5 In NRW hat die neue Landesregierung diese Verordnung zum Schuljahr 2017/18 wieder zurückgenommen, so dass abzuwarten bleibt, wie sich die Anzahl der Förderschulen weiter entwickeln wird. 
| Thomas Kemper \& Janka Goldan

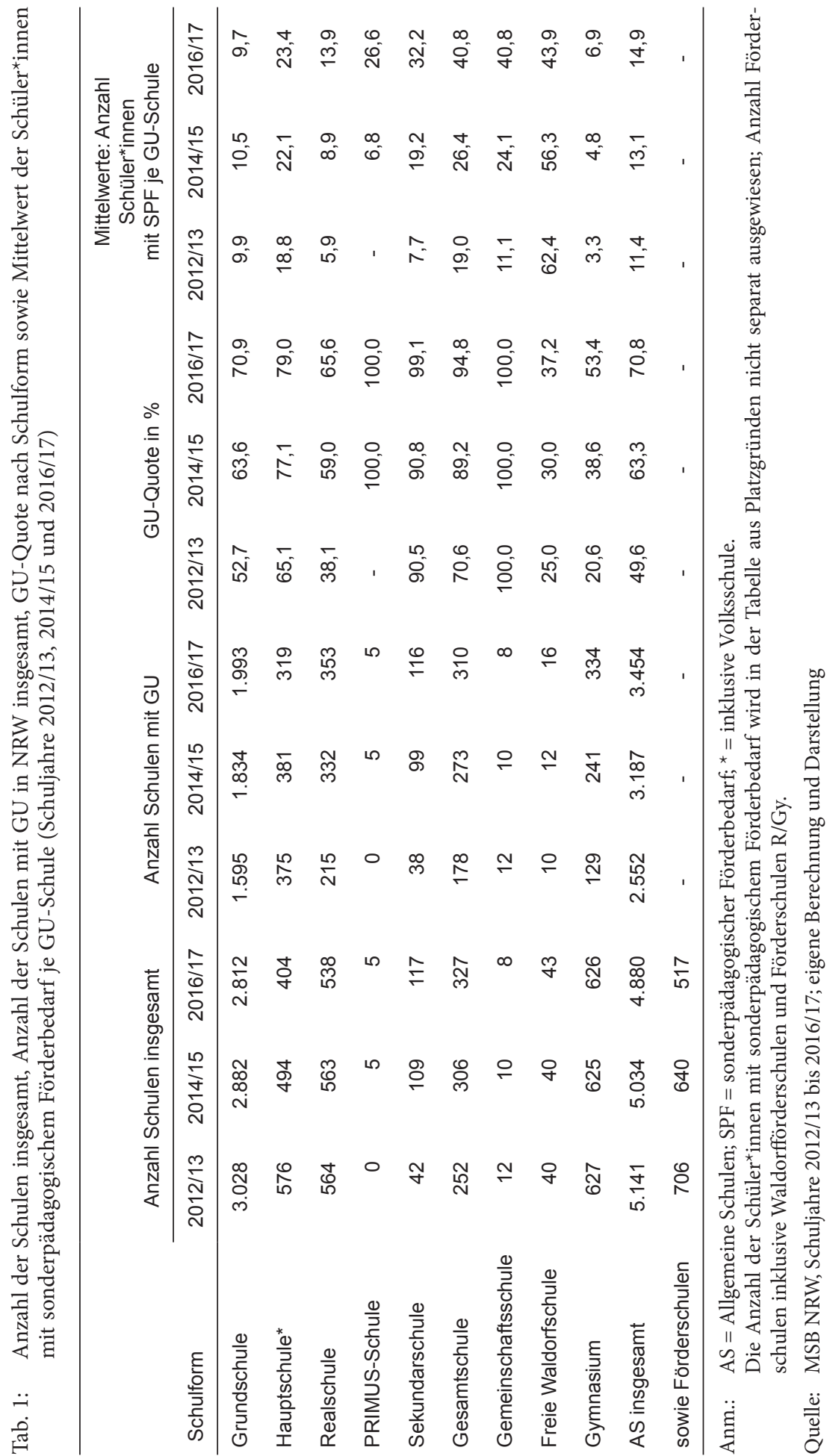


Geistige Entwicklung (GE) $+2,5 \%$, Sehen (SE) $+3,6 \%$; eigene Berechnung, ohne Abbildung). Die Schulstatistik gibt zudem näherungsweise Auskunft zum Hauptförderschwerpunkt von Förderschulen. Die Entwicklung der Anzahl der Förderschulstandorte ist in diesem Zeitraum für fast alle Hauptförderschwerpunkte von deutlichen Rückgängen geprägt (Lernen, Sprache und Emotional-soziale Entwicklung (LSE) $-38,8 \%$, HK -13,3\%, Körperlich-motorische Entwicklung (KM) -2,8\%, GE -0,9\%, SE +/-0,0; eigene Berechnung, ohne Abbildung). ${ }^{6}$

Zwischen den Schuljahren 2012/13 und 2016/17 ist die Anzahl der Schulen mit GU von 2.552 auf 3.454 Schulen angestiegen. Daraus ergibt sich ein kontinuierlicher Anstieg der GU-Quote von 49,6 auf 70,8 Prozent. Somit findet an beinahe drei Vierteln aller Schulen in NRW GU im Sinne der Definition statt. ${ }^{7}$ Differenziert nach Schulform zeigen sich die höchsten Anstiege in der GU-Quote für die Gymnasien und Realschulen $(+159$ bzw. $+72 \%)$, wenn auch ausgehend von einem quantitativ geringen GU-Niveau. Mittlerweile findet an mindestens jeder zweiten Schule dieser Schulformen GU statt.

Die geringsten prozentualen Veränderungen in der GU-Quote sind hingegen für Haupt-, Sekundar-, Gesamt- sowie Gemeinschaftsschulen zu beobachten, die bereits vor der Umsetzung des 9. SchRÄG hohe GU-Quoten aufwiesen und deren GU-Quote im Schuljahr 2016/17 zwischen 79 und 100 Prozent beträgt.

Die in der Tabelle dargestellten Durchschnittswerte der Schüler*innen mit sonderpädagogischem Förderbedarf je GU-Schule zeigen erhebliche Unterschiede zwischen den Schulformen auf. Während eine GU-Grundschule im Durchschnitt von rund 10 Schüler*innen mit sonderpädagogischem Förderbedarf besucht wird, zählen GU-Gymnasien nur knapp 7 Schülerinnen. An Gemeinschafts-, Gesamt- und Waldorfschulen sind es jeweils mehr als 40 Schülerinnen mit Förderbedarf je GUSchule. Tendenziell zeigt sich auf Ebene der Schulen, dass eine höhere GU-Quote auch mit einer steigenden Anzahl an Schüler*innen mit sonderpädagogischem Förderbedarf einhergeht.

Tabelle 2 veranschaulicht, wie die GU-Quote in Abhängigkeit von Förderschwerpunkt und Schulform variiert. ${ }^{8}$

6 Im Rahmen dieses Beitrages wird kein Fokus auf Privatschulen gelegt. Die Datenbasis erlaubt prinzipiell auch eine Differenzierung der Förderschulen nach privater und öffentlicher Trägerschaft. Eine erste schulstatistische Auswertung für das Schuljahr 2016/17 zeigt, dass weniger als 15 Prozent aller Förderschulen in NRW in privater Trägerschaft sind (76 von 517 Förderschulen insgesamt; eigene Berechnung, ohne Abbildung).

7 Die quantitative Operationalisierung von GU lässt keine Rückschlüsse darauf zu, ob aus pädagogischer Sicht in den Klassen tatsächlich GU bzw. inklusiver Unterricht stattfindet.

8 In die nach Förderschwerpunkt differenzierende GU-Quote fließen nur diejenigen Schulen mit ein, die wenigstens einen Schüler bzw. eine Schülerin dieses Förderschwerpunktes im GU aufweisen. 
Thomas Kemper \& Janka Goldan

Tab. 2: $\quad$ GU-Quote nach Förderschwerpunkt und Schulform in NRW (Schuljahr 2016/17)

\begin{tabular}{lrrrrrr}
\hline \multirow{2}{*}{ Schulform } & \multirow{2}{*}{\begin{tabular}{c} 
Anzahl \\
\cline { 3 - 7 }
\end{tabular}} & \multicolumn{5}{c}{ Schulen } \\
\cline { 3 - 7 } & 2.812 & 66,6 & 25,5 & 24,2 & 17,4 & 9,0 \\
\hline Grundschule & 404 & 78,0 & 18,6 & 21,5 & 8,4 & 5,0 \\
Hauptschule* & 538 & 61,0 & 23,4 & 11,0 & 19,3 & 11,9 \\
Realschule & 5 & 100,0 & 80,0 & 60,0 & 40,0 & 20,0 \\
PRIMUS-Schule & 117 & 99,1 & 58,1 & 33,3 & 35,0 & 20,5 \\
Sekundarschule & 327 & 93,3 & 64,2 & 36,4 & 51,7 & 31,5 \\
Gesamtschule & 8 & 100,0 & 50,0 & 37,5 & 50,0 & 25,0 \\
Gemeinschaftsschule & 43 & 34,9 & 9,3 & 11,6 & 7,0 & 2,3 \\
Freie Waldorfschule & 626 & 42,7 & 15,8 & 5,0 & 18,2 & 13,3 \\
Gymnasium & 4.880 & 66,2 & 26,8 & 21,0 & 19,7 & 11,3 \\
\hline insgesamt & & & & &
\end{tabular}

Anm.: $\quad *$ einschließlich Volksschule.

Quelle: MSB NRW, Schuljahr 2016/17; eigene Berechnung und Darstellung

In NRW unterrichten 66,2 Prozent der Schulen Schüler*innen in den Förderschwerpunkten LSE. ${ }^{9}$ Hingegen findet an maximal jeder vierten Schule GU in den Förderschwerpunkten KM, GE, HK oder SE statt (die GU-Quote beträgt hier zwischen 11,3 und 26,8 Prozent).

Werden diese Ergebnisse differenziert nach den quantitativ bedeutsamsten Schulformen betrachtet, dann zeigen sich überdurchschnittliche GU-Quoten für die Sekundar- und Gesamtschulen - und dies unabhängig vom Förderschwerpunkt. Eine überdurchschnittliche GU-Quote ist auch für Hauptschulen festzustellen, allerdings nur im Bereich LSE. In den Bereichen KM, HK und SE fällt die GU-Quote an Hauptschulen nur unterdurchschnittlich aus und beträgt z.T. nur die Hälfte der GU-Quote insgesamt.

Ausnahmslos unterdurchschnittlich fallen die GU-Quoten an Waldorfschulen aus. Für Gymnasien sind erheblich unterdurchschnittliche GU-Quoten für die Förderschwerpunkte GE, LSE und KM auszumachen. Dennoch sind die Ergebnisse bemerkenswert, da sie belegen, dass sich Gymnasien langsam der Inklusion öffnen bzw. öffnen müssen, auch wenn weitere Berechnungen zeigen, dass es sich gemessen an der Gesamtzahl der Gymnasiast*innen um einen relativ geringen Anteil von Schülerinnen mit sonderpädagogischem Förderbedarf handelt $(0,4 \%$, ohne $\mathrm{Ab}$ bildung).

9 Zur Begründung, warum LSE häufig als ein Förderschwerpunkt berichtet wird, vgl. PreussLausitz (2016). 
Weiter sollen die Schülerzahlen an allen Schulen in NRW differenziert nach Schulform für die Schuljahre 2012/13 bis 2016/17 berichtet werden (eigene Berechnung anhand von Daten des Ministeriums für Schule und Bildung (MSB) NRW, ohne Abbildung). Auf Grundlage der absoluten Schülerzahlen wird die Inklusionsquote berechnet (d.h. der prozentuale Anteil derjenigen mit sonderpädagogischem Förderbedarf im GU). Zwischen 2012/13 und 2016/17 ist die Inklusionsquote von 1,5 auf 2,8 Prozent angestiegen. Differenziert nach Schulform zeigen sich im Schuljahr 2016/17 die höchsten Inklusionsquoten an Haupt- und PRIMUS-Schulen (von 8,5 bzw. 8,1\%) sowie an den Sekundar- und Gemeinschaftsschulen (mit 7,4 bzw. 7,6\%). Die geringsten Inklusionsquoten zeigen sich für Realschulen $(2,1 \%)$ und insbesondere für Gymnasien (0,4\%). Somit hat an Realschulen jede 48ste Schülerin bzw. jeder 48ste Schüler einen sonderpädagogischen Förderbedarf, an Gymnasien sogar nur jede ${ }^{\star}$ 227ste. Diese Zahl veranschaulicht in Kombination mit der in Tabelle 1 dargestellten GU-Quote, dass insgesamt an relativ vielen Gymnasien GU im Sinne der Operationalisierung stattfindet. Die niedrige Inklusionsquote belegt allerdings, dass es sich gemessen an der Gesamtzahl der Schüler*innen an Gymnasien eher um Einzelfälle handelt.

In Tabelle 3 werden für NRW weitere inklusionsrelevante Kennzahlen mit Bezug zur Primarstufe und Sekundarstufe I berichtet. Diese werden um die absoluten Zahlen der Schüler*innen an Allgemeinen Schulen und an Förderschulen ergänzt, um die entsprechenden Anteile und Quoten - unter Berücksichtigung des demografischen Trends - besser beurteilen zu können.

Die Anzahl der Schüler*innen insgesamt sowie derjenigen an Allgemeinen Schulen ist bis zum Schuljahr 2015/16 kontinuierlich zurückgegangen. Für das darauffolgende Schuljahr 2016/17 ist erstmalig wieder ein leichter Anstieg der Schülerzahlen erkennbar. Die Zahl der Förderschüler*innen ist im gesamten Zeitraum um 13.200 bzw. um 16 Prozent zurückgegangen. Der Rückgang ist erheblich, auch wenn sich dieser zwischen den Schuljahren 2015/16 und 2016/17 deutlich abgeschwächt hat. Im selben Zeitraum ist die Anzahl der Schüler*innen mit sonderpädagogischem Förderbedarf an Allgemeinen Schulen innerhalb von vier Jahren von 28.800 auf 51.200 (bzw. um $+78 \%$ ) angestiegen. Der Rückgang um 13.200 an den Förderschulen ist begleitet von einem stärkeren Anstieg in den Allgemeinen Schulen um 22.300. Insgesamt ist die Anzahl der Schüler*innen mit sonderpädagogischem Förderbedarf um 8 Prozent auf 122.600 angestiegen.

Dieser absolute Anstieg bildet sich - bei insgesamt zurückgehenden Schülerzahlen auch in der Förderquote ab, die innerhalb von vier Jahren kontinuierlich von 6,5 auf 7,4 Prozent angestiegen ist. Zwischen 2012/13 und 2016/17 hat sich die Inklusionsquote von 1,7 auf 3,1 Prozent annähernd verdoppelt, während die Exklusionsquote von 4,9 auf 4,3 Prozent gesunken ist. Auch hier ist bemerkenswert: Ein Anstieg in den Allgemeinen Schulen um 1,4 Prozent geht mit einem Rückgang 
| Thomas Kemper \& Janka Goldan

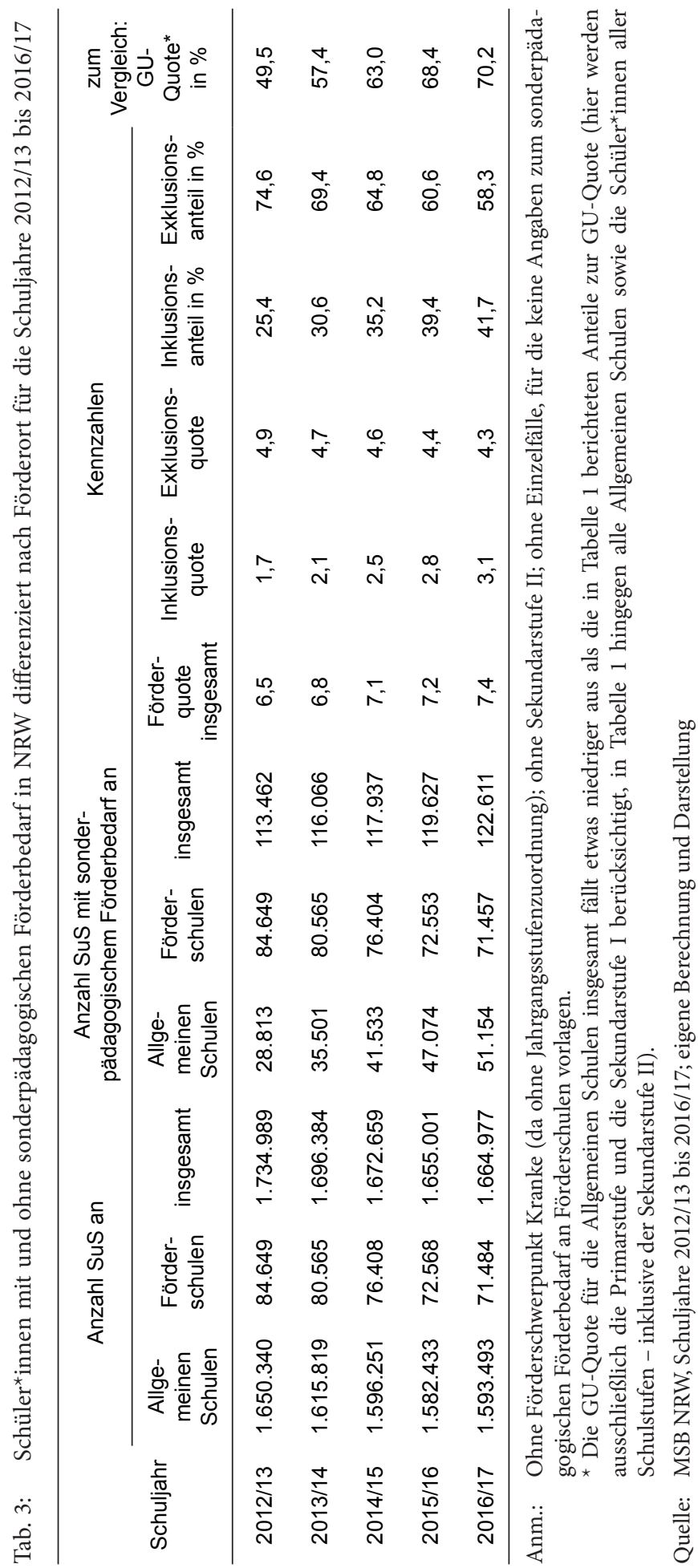


der Schülerzahl um nur 0,6 Prozent in den Förderschulen einher. Die quantitative Verschiebung des Beschulungsortes wird (auch) durch den Inklusions- und den Exklusionsanteil verdeutlicht: Der Inklusionsanteil wächst von 25,4 auf 41,7 Prozent an, was mit einem Rückgang des Exklusionsanteils von 74,6 auf 58,3 Prozent einhergeht. Hierdurch wird zunächst eine deutlich zunehmende inklusive Beschulung innerhalb von wenigen Jahren nahegelegt (bzw. eine zurückgehende separierende Beschulung). Im Anstieg der Förderquote, d.h. der Zahl der Schüler*innen mit festgestelltem sonderpädagogischem Förderbedarf, zeigt sich allerdings auch, dass an den Allgemeinen Schulen die Bereitschaft zunimmt, formale Feststellungsverfahren einzuleiten. In der Literatur werden unterschiedliche Vermutungen zur Erklärung dieser Entwicklung angeführt. Häufig wird dabei auf das „Ressourcen-EtikettierungsDilemma"10 verwiesen (z.B. Hillenbrand, 2013), d.h., dass die Bereitschaft zu formalen Diagnosen im Zuge der Inklusion angestiegen ist, weil jede zusätzliche Schülerin bzw. jeder zusätzliche Schüler im Regelschulsystem in Abhängigkeit von der lokalen Regelung u. U. einen direkten Anspruch auf zusätzliche Ressourcen auslöst. Im Inklusionsanteil spiegelt sich in der Folge ein steigender Trend wider, der ohne Berücksichtigung der Entwicklung von Förder- und Exklusionsquote kaum aussagekräftig ist.

Wie in Tabelle 3 dargestellt, beträgt im Schuljahr 2016/17 die GU-Quote für das Land NRW 70,2 Prozent. Ausgehend von diesem Befund werden in Abbildung 1 die regionalen Disparitäten in der GU-Quote dargestellt. ${ }^{11}$

Die Karte verdeutlicht, dass zwischen den Kreisen und kreisfreien Städten in NRW z. T. erhebliche Unterschiede in der GU-Quote existieren. Es gibt einerseits Kreise, in denen noch nicht einmal an jeder zweiten Allgemeinen Schule GU stattfindet (in zwei Kreisen liegen die GU-Quoten zwischen 40 und 50 Prozent). Diesen stehen Kreise gegenüber, in denen an fast allen Allgemeinen Schulen GU durchgeführt wird (je ein Kreis und eine kreisfreie Stadt weisen GU-Quoten von mindestens 95 Prozent auf). Weiter fällt auf, dass vereinzelt große Unterschiede zwischen direkt aneinander angrenzenden Kreisen bestehen - so beträgt im Nordosten von NRW z. B. in einem Landkreis die GU-Quote 40,2 Prozent; in einem benachbarten Landkreis fällt dieser Anteil mit 80,0 Prozent etwa doppelt so hoch aus. ${ }^{12}$

10 Hierunter wird die steigende Bereitschaft dazu verstanden, einen sonderpädagogischen Förderbedarf zu diagnostizieren, weil hieran Ressourcen (z. B. in Form von Lehrpersonal) geknüpft sind. Das Dilemma besteht darin, dass hierdurch ein Anreiz für Diagnosen besteht, hingegen im Sinne der UN-BRK Etikettierungen vermieden werden sollen.

11 Das primäre Anliegen besteht darin, auf regionale Disparitäten hinzuweisen; daher wird auf die namentliche Nennung der Kreise bzw. kreisfreien Städte verzichtet.

12 Die abgebildeten und beschriebenen regionalen Unterschiede drücken sich auch in weiteren Lagemaßen aus. Neben dem bereits berichteten arithmetischen Mittel für NRW insgesamt betragen der Median der GU-Quote in den Kreisen und kreisfreien Städten 71,6 Prozent, das 1. Quartil 62,4 Prozent, das 3. Quartil 79,7 Prozent, die Standardabweichung 12,3. Das Minimum der GU-Quoten beträgt in den Kreisen und kreisfreien Städten 40,2 Prozent, das Maximum 98,1 Prozent. 
Abb. 1: GU-Quoten sowie Exklusionsquoten differenziert nach Kreisen und kreisfreien Städten in NRW (Allgemeine Schulen, Schuljahr 2016/17) ${ }^{13}$
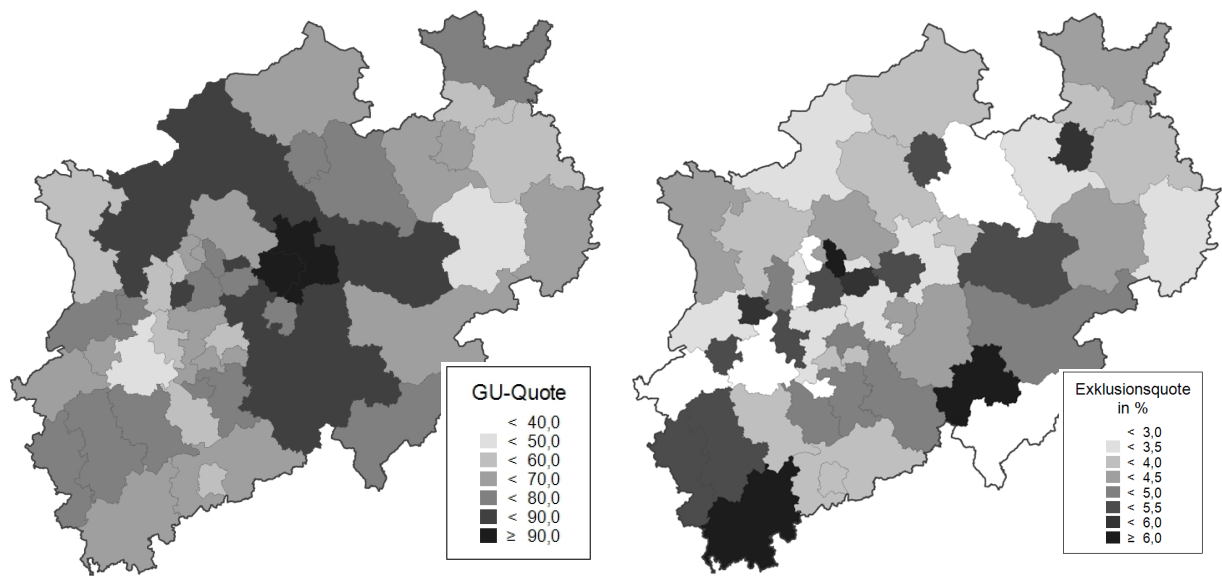

Anm.: Ohne Sekundarstufe II.

Quelle: MSB NRW, Schuljahr 2016/17; eigene Berechnung und Darstellung

Um zu verdeutlichen, wie sich die Anteile der inklusiv beschulten Schüler*innen an GU-Schulen in absoluten Zahlen darstellen, sollen diese punktuell genannt werden: In den Kreisen und kreisfreien Städten variiert die durchschnittliche Zahl der Schüler*innen mit sonderpädagogischem Förderbedarf an Allgemeinen Schulen mit GU zwischen 10,1 und 24,1 (bzw. zwischen 7,6 und 17,8 bezogen auf alle Allgemeinen Schulen - d.h. sowohl mit als auch ohne GU). Bezogen auf alle Allgemeinen Schulen mit GU bleibt weiter festzuhalten, dass etwa ein Drittel (32,7\%) maximal 5 Schüler^innen mit sonderpädagogischem Förderbedarf aufweisen. Mehr als die Hälfte der GU-Schulen $(53,9 \%)$ wird von 10 oder weniger Schülerinnen mit Förderbedarf besucht (eigene Berechnung, ohne Abbildung).

Am Beispiel der Exklusionsquote soll weiter gezeigt werden, dass die inklusionsrelevanten Kennzahlen auf Ebene der Kreise und kreisfreien Städte erheblichen Schwankungen unterliegen. Wie in Tabelle 3 dargestellt, beträgt im Schuljahr 2016/17 die Exklusionsquote für das Land NRW 4,3 Prozent. Um zu untersuchen, ob innerhalb von NRW regionale Unterschiede hinsichtlich der Kennzahl existieren, werden auf der Ebene der Kreise und kreisfreien Städte die Exklusionsquoten berechnet und kartografisch dargestellt.

Die Spannweite zwischen den Kreisen und kreisfreien Städten reicht von 2,1 bis zu 7,2 Prozent. Die höchsten Quoten von über 6 Prozent sind für eine kreisfreie Stadt

13 Das Minimum Allgemeiner Schulen in den Kreisen und kreisfreien Städten beträgt 29, das Maximum beträgt 240, der Mittelwert je Kreis bzw. kreisfreier Stadt 92,1 (Median 89; Standardabweichung 41,0; 1. Quartil: 61,5; 3. Quartil: 120,0). 
und für zwei Landkreise zu beobachten. Insgesamt sieben Kreise und kreisfreie Städte weisen Quoten von unter 3 Prozent auf. Hierunter befinden sich jeweils ein Kreis und eine kreisfreie Stadt mit Quoten von unter 2,5 Prozent. ${ }^{14}$ Auch zwischen direkt aneinander angrenzenden Kreisen bzw. kreisfreien Städten finden sich erneut erhebliche Unterschiede - die größte Differenz besteht zwischen zwei benachbarten kreisfreien Städten und beträgt 3,9 Prozentpunkte.

\section{Fazit}

Anhand von Daten der amtlichen Schulstatistik des Landes NRW wurde gezeigt, wie sich die Entwicklung schulischer Inklusion quantitativ beschreiben lässt. Dazu wurden die allgemein gebräuchlichen Kennzahlen zunächst vergleichend dargestellt, für die Schuljahre 2012/13 bis 2016/17 berechnet und interpretiert: Gemessen am Inklusionsanteil zeigte sich eine deutliche Zunahme der schulischen Inklusion in NRW. Hingegen ist die Exklusionsquote seit 2012/13 bis zum Schuljahr 2016/17 nur um 0,6 Prozent zurückgegangen, während die Förderquote von 6,5 Prozent auf 7,4 Prozent angestiegen ist und sich die absolute Zahl derjenigen mit sonderpädagogischem Förderbedarf an Allgemeinen Schulen fast verdoppelt hat. Diese Befunde indizieren eher einen Anstieg der Diagnosen an Allgemeinen Schulen als einen tatsächlichen Anstieg der schulischen Inklusion, der mit einem deutlicheren Rückbau des Förderschulsystems verbunden wäre.

Insbesondere vor dem Hintergrund des in der UN-BRK verankerten Rechts auf wohnortnahe Beschulung wurde eine neue Kennzahl eingeführt: Die „GU-Quote“ bezeichnet den Anteil der Schulen mit Gemeinsamem Unterricht an allen Allgemeinen Schulen. Sie ist ein Indiz dafür, ob „Menschen mit Behinderungen gleichberechtigt mit anderen in der Gemeinschaft, in der sie leben, Zugang zu einem integrativen [...] Unterricht an Grundschulen und weiterführenden Schulen haben" (Art. 24 Abs. 2 UN-BRK). Die GU-Quote lässt konkrete Rückschlüsse darauf zu, ob sich die Schüler*innen mit Förderbedarf auf viele oder nur wenige Allgemeine Schulen verteilen, d.h., ob ein integratives Schulsystem im Sinne der UN-BRK eingerichtet wird oder ob es im Allgemeinen Schulsystem zu einer weiteren schulischen Separation kommt (Aichele \& Kroworsch, 2017). Damit kann die Kennzahl als sinnvolle Ergänzung zur Exklusionsquote angesehen werden, die auf Basis amtlicher Daten weiter ausdifferenziert werden kann. In Abhängigkeit von der Fragestellung kann es z.B. sinnvoll sein, weiter nach Förderschwerpunkten oder Schulformen zu differenzieren und diese in Beziehung zu anderen inklusionsrelevanten Kennzahlen auf verschiedenen Analyseebenen (z. B. Klassen-, Schulstufen- oder Schulebene) zu setzen.

14 Ergänzt werden sollen weitere Lagemaße, die die regionalen Unterschiede beschreiben. Der Median für die Exklusionsquote in den Kreisen und kreisfreien Städten beträgt 3,98 Prozent, das 1. Quartil 3,42 Prozent, das 3. Quartil 5,07 Prozent, die Standardabweichung 1,1, das regionale Minimum 2,07 Prozent und das Maximum 7,24 Prozent. 
Die anhand von Daten der amtlichen Schulstatistik durchgeführten regionalen Analysen haben exemplarisch gezeigt, dass erhebliche regionale Disparitäten in der Umsetzung der schulischen Inklusion bestehen. Am Beispiel der GU-Quote und der Exklusionsquote wurde verdeutlicht, wie stark die Entwicklung der schulischen Inklusion regional variiert. Diese Tatsache lässt sich einerseits dadurch erklären, dass die unterschiedlichen Schulträger und Schulaufsichten - darunter Kreise, kreisfreie Städte, Gemeinden und Landschaftsverbände - eigene bildungspolitische Entscheidungen treffen. Um die Ursachen genauer zu ergründen, müssten verschiedene Entscheidungsebenen der einzelnen Regionen (Landes- und Bezirksregierung; Schulaufsicht; Schulen) unter Berücksichtigung der besonderen lokalen Gegebenheiten in den Fokus der Untersuchung gerückt werden. Hinsichtlich der Varianz in der Förderquote könnten noch kleinräumigere Analysen (z.B. auf Ebene von Gemeinden, Statistischen Gebieten oder Baublöcken) zeigen, zu welchem Anteil die Disparitäten durch die unterschiedliche (z.B. ethnische und sozioökonomische) Zusammensetzung der Wohnbevölkerung aufzuklären sind.

Die Steuerungsrelevanz der erzielten Ergebnisse ergibt sich u. a. dadurch, dass die Entwicklung der schulischen Inklusion empirisch anhand von aussagekräftigen Inklusionsindikatoren überprüft werden kann. Auch werden z.B. diejenigen Regionen identifiziert, die hinsichtlich der Umsetzung unterdurchschnittlich abschneiden. Dies würde es beispielsweise erlauben, zielgenau finanziell oder gesetzlich nachzusteuern und dadurch Bildung gerechter zu gestalten.

\section{Literatur und Internetquellen}

Aichele, V., \& Kroworsch, S. (2017). Inklusive Bildung ist ein Menschenrecht. Warum es die inklusive Schule für alle geben muss. Berlin: Deutsches Institut für Menschenrechte.

Autorengruppe Bildungsberichterstattung (2014). Bildung in Deutschland 2014. Ein indikatorengestützter Bericht mit einer Analyse zur Bildung von Menschen mit Behinderungen. Bielefeld: Bertelsmann.

Autorengruppe Bildungsberichterstattung (2018). Bildung in Deutschland 2018. Ein indikatorengestützter Bericht mit einer Analyse zu Wirkungen und Erträgen von Bildung. Bielefeld: Bertelsmann.

Brüggemann, C., \& Tegge, D. (2016). Kennziffern in der Kritik: Anmerkungen zur indikatorengestützten Darstellung von Inklusion in der internationalen Bildungsberichterstattung. Zeitschrift für Inklusion, 11 (1). Zugriff am 23.03.2019. Verfügbar unter: https://www.inklusion-online.net/index.php/inklusion-online/article/view/344.

Dietze, T. (2011). Sonderpädagogische Förderung in Zahlen - Ergebnisse der Schulstatistik 2009/10 mit einem Schwerpunkt auf der Analyse regionaler Disparitäten. Zeitschrift für Inklusion, 6 (2). Zugriff am 23.03.2019. Verfügbar unter: https://www.inklusion-on line.net/index.php/inklusion-online/article/view/88/88.

Döttinger, I., \& Hollenbach-Biele, N. (2015). Auf dem Weg zum gemeinsamen Unterricht? Aktuelle Entwicklungen zur Inklusion in Deutschland. Gütersloh: Bertelsmann Stiftung. 
Heimlich, U., \& Wittko, M. (2018, Januar). Sozialräumliche Betrachtung der inklusiven Schullandschaft in Kempten - ein Zwischenbericht (Forschungsbericht Nr. 11). München: Forschungsstelle Inklusionsforschung (F!F) der LMU München.

Hillenbrand, C. (2013). Inklusive Bildung in der Schule: Probleme und Perspektiven für die Bildungsberichterstattung. Zeitschrift für Heilpädagogik, 64 (9), 359-369.

Hollenbach-Biele, N. (2014). Update Inklusion - Datenreport zu den aktuellen Entwicklungen. Gütersloh: Bertelsmann Stiftung.

Kemper, T. (2012). Untersuchungen zum Schulerfolg von Migranten mit sonderpädagogischem Förderbedarf bei separierter und integrierter Beschulung in Rheinland-Pfalz. Zeitschrift für Heilpädagogik, 63 (9), 360-368.

Kemper, T., \& Weishaupt, H. (2011). Zur Bildungsbeteiligung ausländischer Schüler an Förderschulen - unter besonderer Berücksichtigung der spezifischen Staatsangehörigkeit. Zeitschrift für Heilpädagogik, 62 (10), 419-431.

Klemm, K. (2010). Gemeinsam lernen. Inklusion leben. Status Quo und Herausforderungen inklusiver Bildung in Deutschland. Gütersloh: Bertelsmann Stiftung.

Klemm, K. (2013). Inklusion in Deutschland - eine bildungsstatistische Analyse. Gütersloh: Bertelsmann Stiftung.

Klemm, K. (2014). Auf dem Weg zur inklusiven Schule: Versuch einer bildungsstatistischen Zwischenbilanz. Zeitschrift für Erziehungswissenschaft, 17 (4), 625-637. https://doi. org/10.1007/s11618-014-0543-0

Klemm, K. (2015). Inklusion in Deutschland - Daten und Fakten. Gütersloh: Bertelsmann Stiftung.

Klemm, K., \& Preuss-Lausitz, U. (2017). Inklusion in progress. Analysen, Herausforderungen, Empfehlungen (Böll.brief Teilhabegesellschaft \#4). Berlin: Heinrich-Böll-Stiftung.

KMK (Sekretariat der Ständigen Konferenz der Kultusminister der Länder in der Bundesrepublik Deutschland) (2016). Sonderpädagogische Förderung in Schulen 2005 bis 2014. Berlin: KMK.

Kroworsch, S. (2017). Das Recht auf inklusive Bildung. Allgemeine Bemerkung Nr. 4 des UNAusschusses für die Rechte von Menschen mit Behinderungen. Berlin: Deutsches Institut für Menschenrechte.

Lütje-Klose, B., Neumann, P., \& Streese, B. (2017). Schulische Inklusion in Nordrhein-Westfalen (NRW) - sieben Jahre nach Ratifizierung der UN-BRK. Zeitschrift für Inklusion, 12 (2). Zugriff am 23.03.2019. Verfügbar unter: https://www.inklusion-online.net/ index.php/inklusion-online/article/view/416/321.

Malecki, A. (2014). Sonderpädagogischer Förderbedarf - eine differenzierte Analyse. WISTA - Wirtschaft und Statistik, (10), 591-601.

Mißling, S., \& Ückert, O. (2014). Inklusive Bildung: Schulgesetze auf dem Prüfstand. Berlin: Deutsches Institut für Menschenrechte.

Preuss-Lausitz, U. (2016). Throughput instead of Input. Herausforderungen beim Wegfall der Feststellungsdiagnostik in den Förderbereichen Lernen, emotionale und soziale Entwicklung und Sprache. Zeitschrift für Heilpädagogik, 67 (5), 204-214.

Sälzer, C., Gebhardt, M., Müller, K., \& Pauly, E. (2015). Der Prozess der Feststellung sonderpädagogischen Förderbedarfs in Deutschland. In P. Kuhl, P. Stanat, B. Lütje-Klose, C. Gresch, H. A. Pant \& M. Prenzel (Hrsg.), Inklusion von Schülerinnen und Schülern mit sonderpädagogischem Förderbedarf in Schulleistungserhebungen (S. 129-152). Wiesbaden: Springer. https://doi.org/10.1007/978-3-658-06604-8_5

Schneider, K., Klemm, K., Kemper, T., \& Goldan, J. (2017). Dritter Bericht zur Evaluation des Gesetzes zur Förderung kommunaler Aufwendungen für die schulische Inklusion in Nordrhein-Westfalen. Zugriff am 23.03.2019. Verfügbar unter: https://www.wib.uniwuppertal.de/fileadmin/wib/documents/publications/WIB_EvalInklF\%C3\%B6G_3_ Bericht_20170718_final.pdf. 
Weishaupt, H. (2017). Die Förderschule als sonderpädagogischer Förderort. In M. Gercke, S. Opalinski \& T. Thonagel (Hrsg.), Inklusive Bildung und gesellschaftliche Exklusion. Zusammenhänge - Widersprüche - Konsequenzen (S. 39-57). Wiesbaden: Springer VS. https://doi.org/10.1007/978-3-658-17084-4_4

Weishaupt, H. (2018). Sonderpädagogische Förderung im Schuljahr 2016/17 - Situation in Hessen und Rheinland-Pfalz. Schulverwaltung Hessen/Rheinland-Pfalz, 23 (12), 341 346.

Ziegeler, K., \& Schubert, J. (2015). Der Rechtsanspruch auf angemessene Vorkehrungen in der wohnortnächsten inklusiven Schule - eine zu wenig diskutierte internationale Verpflichtung Deutschlands. Gemeinsam leben, 3, 149-155.

Thomas Kemper, Dr. phil., wissenschaftlicher Mitarbeiter am Wuppertaler Institut für bildungsökonomische Forschung (WIB) an der Bergischen Universität Wuppertal.

E-Mail: kemper@uni-wuppertal.de

Janka Goldan, Dipl.-Päd., wissenschaftliche Mitarbeiterin am Wuppertaler Institut für bildungsökonomische Forschung (WIB) und am Institut für Bildungsforschung (IfB) an der Bergischen Universität Wuppertal.

E-Mail: goldan@wiwi.uni-wuppertal.de

Anschrift: Bergische Universität Wuppertal, Gaußstr. 20, 42097 Wuppertal 